\title{
High-Stability Miniature OCXOs Based on Advanced IHR Technology
}

\author{
Igor Abramzon, Sergey Baranushkin, Alexey Gubarev, Oksana Rotova, Vadim Tapkov \\ Magic Xtal Ltd. \\ Omsk, Russia \\ E-mail:mxl@omsknet.ru
}

\begin{abstract}
The paper describes farther progress in the internally heated resonators (IHR) resulting is essential improvement of their frequency stability at remaining extremely small size, power consumption and warm-up time. There is also considered in the paper an effective solution of suppression of the B-mode in the oscillator circuitry based on novel "dynamic selection" method. Experimental study of the OCXOs built on new IHR design proved their excellent temperature stability along with high short-term stability, low aging and phase-noise level. Combined with extremely small size and power consumption it makes new OCXO an effective alternation to conventional OCXOs in a variety of applications.
\end{abstract}

\section{INTRODUCTION}

Being invented more than 30 years ago the IHR had lead to radical reduction of power consumption, volume and warm-up time of the OCXO owing to placing the oven system inside the vacuum volume close to the crystal plate or right on its surface [1]. Originated from the bulky glass holders and simple oven systems providing only 0.1-0.2 ppm frequency stability the IHR design during the past time has evolved into a miniature fully integrated design possessing unsurpassed combination of properties.

From the very beginning the IHR technology was represented by direct the heated resonators (DHR) and the indirect heated resonators (IDHR). In the DHR the crystal plate is heated by the film heaters deposited on its surface that together with usage of stress-compensated SC-cut provides very fast warming-up extremely small size and power consumption.

In the IDHR the crystal plate is placed in a metal can providing its uniform heating that results in essentially better than for DHR temperature stability, although accomplished at bigger sizes and more warm-up time [2].

Further progress in the IHRs was provided by development of the composite heating technology utilizing complex heating the crystal plate: at the start-up - mainly by the film heaters and in the steady state - by the whole power dissipated in the thermo-control system [3]. Such approach accumulating all the advantages of the DHR and IDHR technologies allowed creation of the OCXO providing at about $1.5 \mathrm{ccm}$ volume up to $20 \mathrm{ppb}$ temperature stability, 15 s warm-up time and less than $120 \mathrm{~mW}$ power consumption [4].

However, temperature and short-term stability of these devices was still impacted by the thermal gradients induced in the plate by the film heaters that constrained further development of this technology to utmost stability requirements [5]. As the result, employment of the composite heating resonators was confined to the OCXOs with low and middle stability parameters.

A goal of the present work was development of new IHR design and the oscillator circuitry providing radical improvement of the frequency stability to the level being heretofore a property of high-end conventional OCXO designs.

\section{CONSTRUCTION AND SCHEMATIC PECULIARITIES OF NOVEL OCXO DESIGN}

\section{A. Construction of new IHR}

Construction features of new IHR are outlined in fig.1. It contains a ceramic substrate carrying the whole thermocontroller circuitry including the heating elements and the thermo-sensor. Besides, the substrate is a base for the mounting clips fixing the crystal plate and for the metal screen covering the plate for its uniform heating. The whole micro-oven assembly is arranged inside the TO- 8 holder with excellent insulation from environment by the low conductivity ring and deep vacuum inside the volume.

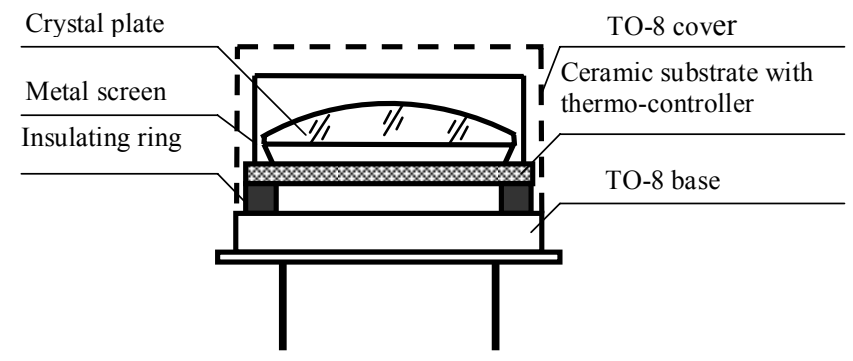

Fig. 1. Schematic drawing of new IHR design.

Owing to perfect insulation of the micro-oven and presence of the metal screen there is reached considerable reduction of the heat currents through the crystal plate and thereby essentially diminished influence of the thermal gradients on its frequency. Together with usage of stresscompensated SC-cut crystal plate that provided sharp improvement of the IHR temperature stability reaching at correct choice of the plate orientation $5 \mathrm{ppb}$ versus $\pm 50^{\circ} \mathrm{C}$ temperature changes. 


\section{B. Schematic methods of the B-mode suppression}

In difference with conventional OCXOs designs in those using the IHR technology the sustaining circuitry is placed out of the heated volume and therefore is subjected to wide temperature variations. Meantime, most common method of the B-mode suppression in SC-cut crystals utilizes LC filtering components being rather temperature sensitive and besides, requiring accurate frequency tuning.

As an effective way of the B-mode suppression there was tested filtering with an AT-cut strip crystal adjusted to the Cmode frequency. Owing to miniature sizes of the strip crystal and essentially better than of the LC components temperature stability, this technique can be successfully used in miniature OCXO constructions providing high temperature stability, although in not too wide temperature range.

A novel method of the B-mode suppression approbated within the work is grounded on different motional parameters of the B and C-modes resulting in essentially different speed of growing their amplitudes after the oscillator switch-on.

The process of setting the oscillation amplitude after applying the input voltage to the circuitry depicted in fig. 2 is governed by the well-known equation:

$$
\frac{d U_{i n}}{d t} \cdot T_{K}+U_{\text {in }} \cdot\left(1-K\left(U_{\text {in }}\right)\right)=0,
$$

where $T_{K}=\frac{2 Q}{\omega_{K}}$ - is the time constant of the oscillator loop; $K\left(U_{i n}\right)$ - is the loop gain.

This differential equation has in the linear case following solution:

$$
U_{i n}(t)=U_{i n 0} \cdot \exp \left(\frac{K-1}{T_{K}} \cdot t\right)
$$

For the equivalent circuit depicted in fig. 2 the loop gain factor can be expressed as:

$$
K=\frac{\text { Sae }}{\omega_{q}{ }^{2} \cdot R_{q} \cdot C_{\text {out }} \cdot C_{\text {in }}}=\frac{K_{0}}{\omega_{q}{ }^{2} \cdot R_{q}},
$$

where $\mathrm{Sae}$ - is the trans-conductance of the active element.

In this equation $K_{0}$ is the factor proportional to the loop gain and dependent on the sustain circuit only.

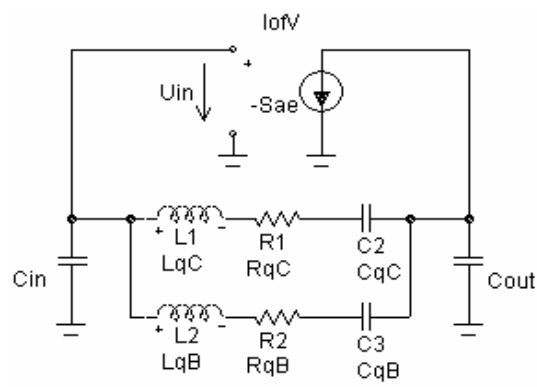

Fig.2. Equivalent circuit of the crystal oscillator.
Out of the equations the process of rise the B and Cmodes amplitudes is described by the expressions:

$$
\begin{aligned}
& U_{B}(t)=U_{B 0} \cdot \exp \left(\frac{\frac{K_{0}}{\omega_{K B}{ }^{2}}-R_{q B}}{2 L_{q B}} \cdot t\right), \\
& U_{C}(t)=U_{C 0} \cdot \exp \left(\frac{\frac{K_{0}}{\omega_{K C}{ }^{2}}-R_{q C}}{2 L_{q C}} \cdot t\right),
\end{aligned}
$$

where $R_{q B}, L_{q B}$ and $R_{q C}, L_{q C}$ are motional parameters of the $\mathrm{B}$ and $\mathrm{C}$ modes accordingly.

As it follows from above expressions, at sufficiently high value of the gain factor $K$ (or $K_{0}$ ) the motional inductance becomes a determinant factor of the amplitude rise rate. Therefore, due to lower value of inductance of the C-mode in SC-cut crystals (about 0.75 of the B-mode inductance) it grows faster than the B-mode reaching the stationary state first and suppressing further development of the latter.

Owing to absence of additional filter elements in the circuitry the "dynamic selection" doesn't contribute into the oscillator size and temperature instability and therefore can be a power solution in many cases.

\section{Construction of the IHR-OCXOs}

Considered above constructive and schematic solutions have lead to creation of a new OCXO design with sizes, in fact, not exceeding the TO- 8 packaging. Two versions of the OCXO construction are depicted in fig. 4 . Both contain TO-8 packaged IHR mounted on the miniature PC board carrying the oscillator circuitry and acting as an adaptor compatible with DIP8 or DIP14 pins-out.

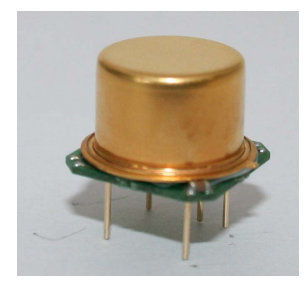

(a)

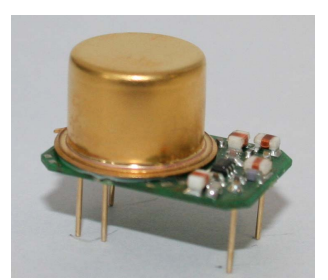

(b)
Fig. 4. The views of the IHR-OCXO designs: (a) DIP8 compatible; (b) DIP14 compatible.

If necessary, the IHR-based oscillator can be easily implemented in any standard OCXO packaging. 


\section{BASIC PERFORMANCE OF THE OCXOS BASED ON NEW IHR TECHNOLOGY}

\section{A. Temperature stability}

As it was considered above new IHR design provides minimizing the thermal gradients in the plate reducing, thereby, their influence on the IHR frequency. Together with novel methods of the B-mode suppression that has resulted in considerable improvement of the OCXO temperatures stability. The fig. 5 illustrates utmost temperature stability results achieved with the $10 \mathrm{MHz}$ OCXOs employing the IHR technology in comparison with best temperature stability of conventional OCXOs.

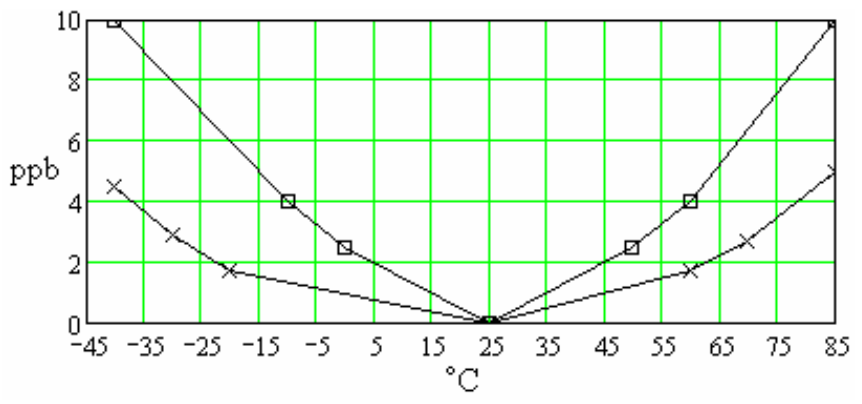

Fig. 5. Utmost temperature stability of OCXOs using conventional (xxx) and the IHR technology $(\square \square \square)$.

As one can see, the best stability attained with the IHR technology reaches $10 \mathrm{ppb}$ in $(-40+85){ }^{\circ} \mathrm{C}$ range and $5 \mathrm{ppb}$ in $(-10+60){ }^{\circ} \mathrm{C}$. Although these figures somewhat yield to the stability of the best conventional OCXOs (obviously due to temperature stabilization of the sustaining circuitry in the latter), they, however, are typical for high-end OCXO designs.

\section{B. Short-term stability and phase-noise}

The STS of the oscillators utilizing the DHR technology was limited by significant influence on their frequency of fluctuations of the thermal stresses induced in the plate by variation of the power in the film heaters caused mainly by the noise in the thermo-control system [5].

In difference with the DHR, in new IHR design the crystal plate is thermally separated from the heaters by the mounting fixture and the ceramic substrate that reduces transmission of the heating power variations into the plate and results in considerable improvement of the OCXO shortterm stability. The fig.6 illustrates Allan variance of the OCXOs utilizing the conventional and the IHR technologies for $0.1 \mathrm{~s}, 1 \mathrm{~s}$ and $10 \mathrm{~s}$ average times.

Out of the plots, based on the IHR technology OCXOs provide Allan variance ranging from about $2 \times 10^{-12}$ to $7 \times 10^{-12}$ at $0.1-1 \mathrm{~s}$ and $10 \mathrm{~s}$ overage time respectively. These values are a bit higher than utmost figures obtained with conventional OCXOs $\left(1.5 \times 10^{-12}\right.$ at $1 \mathrm{~s}$ and $5 \times 10^{-12}$ at $\left.10 \mathrm{~s}\right)$ that can be explained by essentially higher thermal inertia of the conventional oven compared to the micro-oven system of the IHR design.

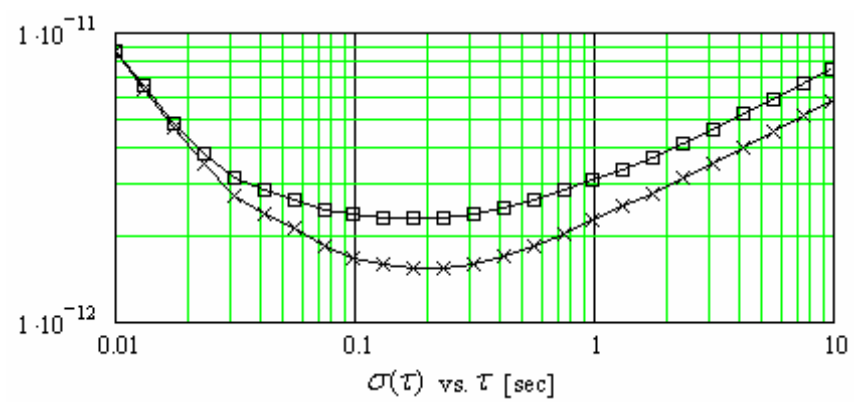

Fig. 6. Allan variance of the OCXOs built with the conventional (xxx) and the IHR technologies $(\square \square \square$ ).

Nevertheless, Allan variance values attained with the IHR technology are typical for the high-end OCXOs. That allows wide application of new OCXOs excepting those cases when extra-high STS is a must.

The phase-noise pattern typical for the IHR-based OCXOs is displayed in Fig. 7. As one can see, the phasenoise level accomplished with the new oscillators (-100 $\mathrm{dBc} / \mathrm{Hz}$ at $1 \mathrm{~Hz}$ and of $-165 \mathrm{dBc} / \mathrm{Hz}$ at $10 \mathrm{kHz}$ offset) in fact corresponds to typical figures attained with conventional OCXO designs.

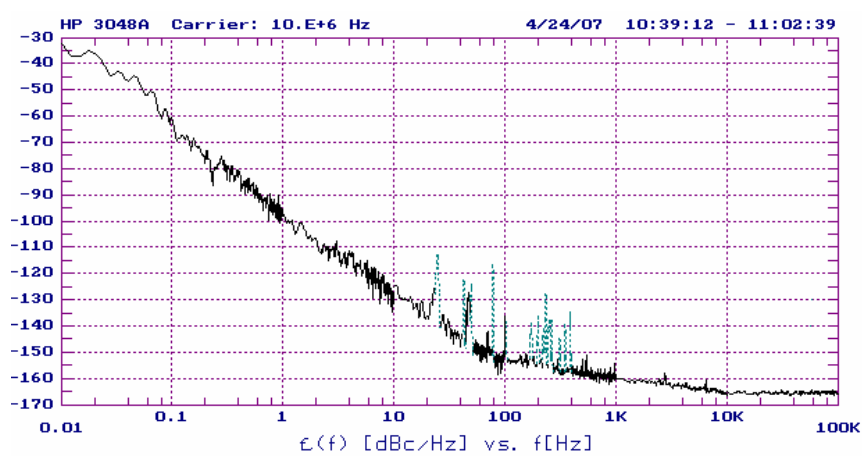

Fig. 7. Typical phase-noise for best IHR-based OCXOs.

\section{Long-term stability}

Long-term stability of the IHR-based oscillators was studied on a group of $10 \mathrm{MHz} 3 \mathrm{~d}$ overtone units during a few months. The test results are represented in fig. 8 as the statistical distribution of the aging figures after 30 days and 60 days of operation.

As it follows from the histogram, about $90 \%$ of the tested units exhibits below $0.5 \mathrm{ppb} /$ day rate after first 30 days operation and $100 \%$ of them - after 60 days. Besides, about half of the units shown less than $0.3 \mathrm{ppb} /$ day drift as early as after first month of the tests. 
Obtained results, are typical for high-end conventional OCXOs that obviously proceeds from similar crystal geometry and the vacuum processes involved in manufacturing of the conventional and the internally heated resonators.

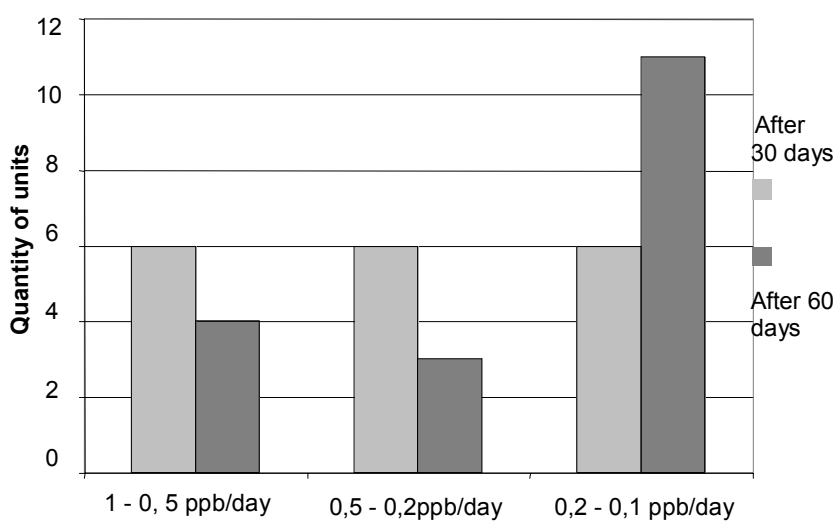

Fig. 8. Aging of the IHR-based OCXOs after 30 and 60 days operation

The table below summarizes main parameters of the best $10 \mathrm{MHz}$ OCXOs utilizing the IHR and conventional technologies.

TABLE. COMPARATIVE PROPERTIES OF THE IHR-BASED AND CONVENTIONAL OCXOS.

\begin{tabular}{|l|c|c|}
\hline \multicolumn{1}{|c|}{ Parameters of the OCXOs } & \multicolumn{2}{|c|}{$\begin{array}{c}\text { Utmost values achieved } \\
\text { with different OCXO } \\
\text { designs }\end{array}$} \\
\cline { 2 - 3 } & $\begin{array}{c}\text { Based on } \\
\text { the IHR } \\
\text { technology }\end{array}$ & $\begin{array}{c}\text { Based on } \\
\text { conventional } \\
\text { technology }\end{array}$ \\
\hline $\begin{array}{l}\text { Temperature stability } \\
\text { over }(-40+85)^{\circ} \mathrm{C} \text { range, ppb }\end{array}$ & 10 & 5 \\
\hline Allan variance at $\tau=1 \mathrm{~s}, 10^{-12}$ & 3 & 2 \\
\hline $\begin{array}{l}\text { Phase-noise }(\mathrm{dBc} / \mathrm{Hz}) \text { at: } \\
1 \text { Hz offset } \\
10 \mathrm{~Hz} \text { offset } \\
10 \mathrm{kHz} \text { offset }\end{array}$ & -102 & -105 \\
\hline $\begin{array}{l}\text { Aging per day after } 30 \mathrm{day} \\
\text { operation, ppb }\end{array}$ & -132 & -135 \\
\hline $\begin{array}{l}\text { Power consumption at } 20^{\circ} \mathrm{C} \\
\text { ambient temperature, } \mathrm{W}\end{array}$ & 0.12 & 0.2 \\
\hline $\begin{array}{l}\text { Warm-up time to } 0.1 \mathrm{ppm} \\
\text { frequency accuracy, s }\end{array}$ & 35 & 1.2 \\
\hline Volume, ccm & 1.5 & 8 \\
\hline
\end{tabular}

As it follows from the data, the oscillators based on new IHR design exhibit the temperature stability, STS, phasenoise and aging rate close to that of high-end conventional OCXOs. At the same time, essential advantageous of the new technology are much smaller OCXO sizes, lower power consumption and faster warming-up.

\section{Conclusion}

1. The new IHR design realizing indirect method of heating the crystal plate in the TO- 8 vacuum holder allowed considerable reduction of the thermal gradients in the plate resulting in radical improvement of the IHR temperature and short-term stability.

2. Novel schematic solutions of the B-mode suppression based on usage of the strip filtering crystal and of the "dynamic selection" method have provided minimal size of the sustaining circuitry and its high stability versus wide temperature changes.

3. The new IHR design and novel schematics solutions have provided creation of a miniature OCXO with superb frequency stability being previously a property of only highend conventional OCXOs.

4. Unsurpassed combination of very high frequency stability with extremely small sizes, low power consumption and short warm-up time makes the new OCXO an effective solution for a variety of applications - from replacement of high-end TCXOs to the fields occupied until now by highstability conventional OCXOs.

\section{REFERENCES}

1. F.G.Tinta, A.S.Matistic, and G.A.Lagasse, "The Direct Temperature Control of Quartz Crystals in Evacuated Enclosures", Proc. of the $24^{\text {th }}$ Annual Symposium on Frequency Control, USA, 1991, pp. 447-451.

2. Ya.L.Vorokhovsky and B.G. Drakhlis. "High-stability Quartz Oscillators on Internally Heated Quartz Resonators with AT and SC Cuts", Proc. of the $45^{\text {th }}$ Annual Symposium on Frequency Control, USA, 1991, pp.447-451.

3. I. Abramzon, A.Dikidzhi, "Improvement of Characteristics of Quartz Resonator-Thermostats with Direct Heating Piezoelement", Proc. of 1992 IEEE Frequency Control Symposium, pp.499-504.

4. I. Abramzon, R.Boroditsky, "Long-Term Stability (Aging) of Evacuated Hybrid OCXO, Proc. of 2001 IEEE International Frequency Control Symposium and PDA Exhibition, pp. 786-789.

5. I. Abramzon, R.Boroditsky, V.Tapkov, "Short-Term Stability of Miniature Double Oven Crystal Oscillators Using Conventional and DHR Technology", proc. of 2003 IEEE International Frequency Control Symposium and PDA Exhibition Jointly with $17^{\text {th }}$ European Frequency and Time Forum, pp.458-463. 